\title{
Espíritu innovador del emprendedor como estrategia de competitividad
}

\section{Innovative spirit of the entrepreneur as a competitiveness strategy}

Tania Patricia Alaña Castillo

Sandy Jahaira Gonzaga Añazco

Andreina Inés González Ordoñez

Universidad Metropolitana del Ecuador, Ecuador

Autor para correspondencia: sandyjgonzagaa; talana@umet.edu.ec; andreinagonzalez09@gmail.com Fecha de recepción: 05 de Julio de 2017 - Fecha de aceptación: 12 de Agosto de 2017

Resumen: El propósito de este artículo es mostrar como el espíritu innovador del emprendedor es una estrategia clave de competitividad empresarial que permitirá un crecimiento sostenible en las micro pequeñas y medianas empresas conocidas como Mipymes, logrando así el progreso económico y la generación de empleo en el país. En ese contexto un elemento clave de la innovación es el empresario emprendedor, siendo la persona que realizará dentro del entorno organizacional las innovaciones en los productos y/o servicios ya sean nuevos o existentes, Por lo tanto, surgen las siguientes interrogantes ¿El espíritu innovador permitirá el desarrollo empresarial?, ¿El emprendimiento es una estrategia de competitividad empresarial?, ¿Cómo logran los microempresarios desarrollar la competitividad mediante el emprendimiento?. Podemos concluir que el emprendedor con espíritu innovador impulsará la craeción de valor mediante la motivación, el conocimiento, la creatividad, la tecnología y por supuesto la innovación que lo impulsará a crear proyectos sociales, económicos, empresariales entre otros, generando rentabilidad a las empresas y al estado. En este documento se consideran los componentes de una vida emprendedora en el entorno empresarial en el que se desarrolla y se analiza como el mismo influye en el espíritu emprendedor del empresario.

Palabras Clave: espíritu innovador; emprendimiento; competitividad; empresa

\begin{abstract}
The purpose of this article is to show how the innovative spirit of the entrepreneur is a key strategy of business competitiveness that will allow sustainable growth is small and medium enterprises known as Mi pymes. Thus achieving economic progress and job creation in the country. In that context, a key element of innovation is the entrepreneur entrepreneur entrepreneur, being the person who will perform within the organizational environment innovations in products and/ or services, whether new or existing. Therefore, the following questions arise: ¿Will the innovative spirit allow business development? ¿Is entrepreneurship a business competitiveness strategy? ¿How do microentrepreneurs achieve competitiveness throught entrepreneurship?, We can conclude that the entrepreneur with an innovative spirit will drive value creation through motivation, knowledge, creativity, technology and of course innovation that will drive it to create social, economic and business projects among others, generating profitability for companies and To the state. This document considers the components of an entrepreneurial life in the business environment in which it is developed and analyzed as it influences the entrepreneurial spirit of the entrepreneur.
\end{abstract}

Key words: innovative spirit; entrepreneurship; competitiveness; company 


\section{Introducción}

Entre las estrategias competitivas implementadas por las empresas de categoría Pymes o Mipymes está el emprendimiento innovador y creativo siendo la actividad que involucra la creación de nuevas ideas de negocios, procesos que permiten transformar la realidad empresarial, expandir productos a mercados internacionales entre otros aspectos claves. Según (Aaron Sabbagh \& Matías Mackinlay, 2011) manifiesta que el empresario innovador es un explorador que encuentra las oportunidades creativas cuando sale de su entorno empresarial a observar cómo los clientes utilizan sus productos y servicios, cuáles son los problemas que enfrentan, y que necesidades poseen.

Por lo tanto, las actividades empresariales son consideradas como un factor determinante para el crecimiento económico y la competitividad de los países (Reinoso \& Uribe, 2013), en este sentido; podemos decir que el emprendimiento empresarial se relaciona con el crecimiento económico, la dinámica productiva de los paises y el empoderamiento de una cultura empresarial, en la actualidad emprendimiento se considera sinónimo del término espíritu empresarial.

Los seres humanos con espiritu emprendedor generan desde la gestión empresarial estrategias que permitan una ventaja competitiva como la innovación creativa (Sabbagh \& Mackinlay 2011) siendo una compentencia individual y colectiva mediante la cual se generan ideas capaces de convertirse en proyectos sustentables y rentables. Por lo tanto, las organizaciones están inmersas en una era de constantes cambios, tales como, cambios en las políticas gubernamentales, en la tecnologia, en la conformación sociocultural, en la cultura empresarial, en este sentido; demandan la implementación de soluciones creativas que superen los patrones habituales de desempeño para maximizar la rentabilidad y la sustentabilidad, mejorando la calidad de vida de los trabajadores y logrando ventajas competitivas.

La cultura es entendida como conjunto de hábitos, normas, creencias, ideologías, valores y experiencias que comparten los individuos dentro de la empresa, por lo tanto, la cultutra del emprendimiento en las organizaciones debe iniciarse con la formación del capital humano de las áreas funcionales técnicas, operativas y administrativas promoviendo en los colaboradores un espíritu emprendedor, entendido como la capacidad de innovar servicios y bienes de manera creativa.

El innovador logra implementar sus ideas cuando sale del proceso de especulación y pone a prueba las mismas, logrando así, generar un resultado que beneficie a la empresa, la economía y a la sociedad. El presente trabajo recoge una revisión teórica de 15 autores en donde se abordan la parte conceptual del espíritu emprendedor y la relación con su entorno empresarial, cuyas actividades están catalogadas como Mipymes, además de la argumentación sobre como la herramienta clave de la competitividad empresarial es la innovación de los productos y/o servicios tanto nuevos o actuales generando valor en las organizaciones, crean fuentes de trabajo, aumentan la rentabilidad tanto de las empresas como del estado.

\section{El espíritu innovador del emprendedor}


Al abordar el tema sobre el espíritu innovador del empresario, hay que empezar explicando las variaciones del fenómeno emprendedor, las primeras investigaciones se centraron en las discrepancias personales que distaban de los que son emprendedores de aquellos que no lo son. Pero dada la variedad y complejidad de la realidad emprendedora, los resultados no fueron fructíferos.

(Gartner, 1985) Considera que la complejidad y variedad de los emprendedores hizo imposible lograr caracterizar a un emprendedor promedio. Sin embargo, los investigadores (Bygrave W. \& Hofer C., 1991) exponen que la delimitación del campo de conocimiento del espíritu emprendedor se amplíe y que además del estudio de las características personales y las funciones del emprendedor incluya el estudio del proceso emprendedor, otro aporte de las investigaciones de Gartner (1985) se enfoca en ciertos elementos esenciales del estudio del emprendedor: a) el proceso, b) el entorno, c) La organización creada, y d) las características del individuo, en este sentido, se podría deducir que existe una relación entre el espiritu innovador y el entorno organizacional.

Según Duarte (2013) la palabra emprendedor proviene del francés entrepreneur (pionero) mencionada desde el siglo XVIII, siendo utilizada inicialmente para referirse a los aventureros tales como Cristóbal Colón quien guiado por una idea orientada al descubrimiento del Nuevo Mundo sin saber con certeza que esperar, en la actualidad esa actitud hacia la incertidumbre es lo que define al emprendedor, autores como (Pérez, Oliver, Merritt, Márquez, León, 2006) consideran que el emprendedor también se caracteriza por comprar productos a precios conocidos para venderlos a precios desconocidos llamados creadores de empresas.

Por lo tanto, en las empresas comenzaron a asociar la asignación de recursos y los riesgos inherentes del negocio al término emprendedor, en este sentido se puede deducir que el término fue ligado a empresarios innovadores. Sin embargo, son las investigaciones de Schumpeter, (1982) las que terminan por refinar el concepto, y rompe de forma radical con la economía tradicional al postular al desequilibrio dinámico producido por el empresario innovador como centro de la teoría económica. Por lo tanto, el concepto de empresario innovador es autónomo de la economía clásica e incompatible con ella, ya que no puede exponer al empresario innovador bajo los esquemas tradicionales, porque tiende a considerarlo como un factor exógeno, tal y como se ve el gobierno, al clima, la guerra, la política y la tecnología, incluso Marx en su libro la teoría de la economía citado por (Drucker, 1985), quien dio una gran importancia a la tecnología, nunca vislumbró al empresario innovador dentro de su sistema económico.

Todo emprendedor inicia con el deseo de convertirse en empresario, esta aspiración es resultado de varios estímulos personales, culturales y sociales, según el autor (Malagón, 2003) considera que todo emprendedor tiene una motivación, dificultad o necesidad ya sea personal, social o familiar, que lo inspira a iniciarse en un proyecto de ámbito económico, político, social, cultural entre otros.

El espíritu emprendedor, entrepreneurship en inglés, se puede entender como la capacidad de una persona para desarrollar proyectos innovadores con el propósito de obtener un beneficio; modificando una idea existencia o desarrollando la creatividad al proponer un producto o servicio nuevo; generando beneficios económicos, sociales o políticos, por otra parte, 
se conoce como espíritu de empresa a la habilidad de la persona por convertir las ideas en hechos, está relacionado con la creatividad, la flexibilidad, la innovación, la planificación, capacidad de asumir riesgos, dinámico y por último la gestión de proyectos con el fin de lograr los objetivos empresariales.

Por otra parte según Castillo (1999) define el termino de espíritu innovador emprendedor como la capacidad de iniciar y operar empresas nuevas, sin embargo, hay miles de negocios, firmas consultoras y tiendas de ropa que previsiblemente han tratado de ser innovadoras, de crecer y de mostrar otras características que demuestran la existencia de espíritu emprendedor en un sentido dinámico, pero han fracaso.

El éxito de un negocio continuamente depende de la importancia de innovar, la creación de cosas nuevas a cargo de gente brillante es la diferente entre un líder y un seguidor. Según Valencia (2017) considera que la integración de equipos propios bien entrenados y dedicados exclusivamente a la busqueda de nuevos productos o servicios, además de la generación de una cultura organizacional fuerte es lo que garantizará la competitividad de las empresas, mediante la innovación siendo está una herramienta clave de crecimiento.

Pronaca empresa que se encuentra en el Ecuador tiene más de 50 colaboradores trabajando directamente en las áreas de Investigación y Desarrollo, la misma ha organizado dentro de sus áreas divisiones de Consumo Humano, de Negocios Agrícola y Pecuario, las cifras señalan que al año la primera división colocó en el mercado entre 15 y 35 innovaciones, sin embargo; Diego Pico director de Investigación y Desarrollo, explica que las nuevas ideas proviene del talento humano, del constante monitoreo del mercado, o de las tendencias que se perciben a nivel mundial.

Por lo tanto el empresario con espíritu innovador es aquel que desarrollará la innovación en las organizaciones siendo una herramienta principal de competitividad, generando rentabilidad y sostenibilidad en la misma.

\section{El emprendimiento cómo estrategia de competitividad empresarial}

Los emprendedores son los principales agentes de cambios en la sociedad, Según el Censo Nacional Económico (2010) el 99,9\% de los establecimientos económicos de la provincia de El Oro son Mipymes (pequeñas y medianas empresas), lo común entre los emprendedores de estas empresas es que originan cambios, producen variedad de oportunidades de trabajo y sirven como modelo para inspirar una nueva generación de emprendedores que promuevan el desarrollo local y global, consiguiendo recompensas para sí msimos y sus familias, y para quienes participan en su proceso, además a los inversionistas que los respaldan.

Según la investigación realizada por Cuervo, Ribeiro, Roig (1999) consideran que la creación de riqueza y el dinamismo de un país se sustenta sobre mediante la competitividad de sus negocios y la misma depende fundamentalmente de las habilidades de sus administradores, la actividad principal de los empresarios emprendedores es la creación empresarial y ésta se puede estudiar a nivel del fortalecimiento de una cultura organizacional. 
Existen diferentes teorias sobre el emprendimiento empresarial, las investigaciones de Schumpeter (1982) se basa en los emprendedores que generan el cambio de una situacion existente a otra, es decir, generan grandes innovaciones y cambios sistémicos que aumentan o crean incertidumbre y promueven el desarrollo de nuevos procesos para crear y/o aumentar la brecha entre líderes y seguidores, los procesos de gestión no son aplicables en esta escuela.

Sin embargo, existe la escuela Austríaca propuesta por el autor (Mises, 1961) quien consideró que el elemento emprendedor está presente en todas las actividades humanas debido a las incertidumbres presentes, y no limitado a un tipo particupar de personas, además Mises, sostiene que el emprendedor es aquel que responde a las señales del mercado con respecto a precios, ganancias y pérdidas, por lo tanto, debido a estas acciones, el emprendedor ayuda a nivelar la demanda y la oferta y, si ha especulado correctamente genera una rentabilidad, de lo contrario, asume las pérdidas por su decisión errónea, en este sentido, se podría entener que los emprendedores generan cambio dentro de una situación existente, aumentan el conocimiento de la situación, reducen el nivel general de incertidumbre a lo largo del tiempo y promueve procesos de mercados que ayudan a reducir o eliminar la brecha existente entre los líderes y los seguidores. Malagón (2003) considera que los emprendedores crean valor al compartir la responsabildiad de desarrollar prácticas nuevas que podrían resolver alguna dificultad existente en la sociedad.

\section{Desarrollo de la competitividad mediante el emprendimiento}

En el documento presentado por la vicepresidencia de la República (2015) denominado Estrategia Nacional para el Cambio de la Matriz Productiva, en el numeral 4.5 componentes de la estrategia considera a la innovación como eje de competitividad empresarial, el documento menciona que El cambio de la matriz productiva requiere de un proceso dinámico de largo plazo. En el período de gobierno entre 2014 y 2017 se pretende iniciar la transición, dejar cimentadas las bases de esa transformación con metas, que consideren los objetivos planteados. La estrategia supone una intervención en tres dimensiones: a) Entorno y competitividad sistémica, b) Desarrollo de un conjunto de cadenas productivas priorizadas c) Impulso a las industrias básicas, Jorge (2017) en su artículo recalca que el plan gubernamental de desarrollar industrias con mayor valor agregado tiene hasta el momento un resultado ambiguo, la intención de la vicepresidencia es generar competitividad desde las empresas, ¿Cómo hacerlo?

Considerando el sistema de desarrollo emprensarial que tiene como componentes las condiciones sociales y económicas, los aspectos culturales, la estructura dinámica y productiva, los mercados de factores, las redes - sociales, los aspectos personales y las regulaciones políticas en la publicación del London Business School (2005) menciona algunas variables específicas del entorno empresarial que influyen en el empredimiento: tales como; acceso financiero, políticas gubernamentales, programas gubernamentales de apoyo, educación y capacitación, transferencia de investigación y desarrollo, infraestructura legal y comercial, apertura del mercado interno, acceso a la infraestrucutra física y las normas socioculturales.

El entorno general en que se desenvuelven las empresas se puede considerar las variables económicas, político-legal, sociocultural, tecnológicas, demográficas y otras variables como las medioambientales, los emprendedores deben comprender los efectos de estas variables de acuerdo a la realidad de cada país y de cada sector donde se quiera promover actividades de 
emprendimiento, sin embargo; existen factores limitantes que no permiten el desarrollo de los mismos, tales como: a) Reducido tamaño de los mercados internos, b) Bajo potencial de crecimiento de los mercados domésticos, c) Escasa capacidad adquisitiva de la población, d) Escasa educación y formación para el emprendimiento, d) Baja posibilidad de realizar investigación y desarrollo, e) Restricciones para acceder a fuentes de financiamiento, f) Obstáculos para la formalización empresarial, g) Deficiente políticas gubernamentales y por último altas cargas impositivas.

Gartner (1985) propone que para estimular la creación de empresas se debe considerar doce factores: a) disponibilidad de capital de riesgo, b) la experiencia de empresarios con experiencia, c) la existencia de fuerza de trabajo calificada, d) facilidades para conseguir proveedores, e) accesibilidad a consumidores y/o nuevos mercados, f) apoyos gubernamentales, g) proximidad con universidades, h) disponibilidad de terrenos e instalaciones, i) acceso a medios de transporte, $\mathrm{j}$ ) actitudes propicias en la población circundante, $\mathrm{k}$ ) disponibilidad de servicios de soporte y condiciones de vida favorable. El Censo Nacional Económico INEC (2010) informa que el desarrollo de los negocios en el país se está consolidando, ya que la microempresa, la pequeña y mediana empresa están en aumento gracias a los emprendedores ecuatorianos, existen 300.000 microempresas que van desde negocios unipersonales a negocios familiares dinámicos.

En el Ecuador existen emprendimientos de éxitos como Consorcio Nobis quien considera que los factores claves del éxito son la creatividad, comunicación, etica, liderazgo, visión y la ejecutabildiad.

\section{Conclusiones}

Las Mipymes en la actualidad deben ser consideradas como motor de crecimiento y generador de empresas, las mismas son creadas desde el espíritu emprendedor, sin embargo el crecimiento de las mismas no es progresivo porque existen limitantes en el desarrollo de los emprendimientos siendo entre otros los más importantes las restricciones para acceder a fuentes de financiamiento, deficientes políticas gubernamentales, entre otros.

Se puede concluir que el empresario con espíritu emprendedor al diseñar e implementar estrategias orientadas a la innovación generan competitividad empresarial, logrando así mejorar la calidad de vida de sus colaboradores, incremento de la rentabilidad en la empresa y desarrollo del estado.

\section{Agradecimiento}

El presente trabajo de investigación forma parte de los resultados del proyecto de investigación "Propuestas de mejoras de la competitividad interna/empresarial de la mipyme de la Provincia de El Oro", de la Carrera Gestión Empresarial de la UMET, Sede Machala, por lo cual se agradece a la UMET por hacer posible presentar esta ponencia.

\section{Bibliografía}

Aarón Sabbagh \& Matías Mackinlay, (2011). El método de la innovación creativa. Buenos Aires: Ediciones Granica S.A. 
Bygrave W. \& Hofer C. (1991). Theorizing about entrepreneurship. Entrepreneurship Theory and Practice, Vol. 16, no.2, pp. 13-22.

Castillo, A. (1999). Estado del Arte en la Enseñanza del Emprendimiento. Intec Chile, 1-21.

Censo Nacional Económico INEC. (2010). Observatorio de la PyME.

Cuervo, Álvaro, Ribeiro Domingo, Roig Salvador. (1999). Entrepreneurship: conceptos, teoría y perspectiva. Introducción. En Entrepreneurship (págs. 1-21). España.

Drucker, P. (1985). Innovation and Entrepreneurship: Practice and Principles. New York: Collins.

Duarte, J. E. (2013). Emprendedor Hacia un emprendimiento sostenible. Colombia: Alfaomega Colombia S.A.

Gartner, W. (1985). A Conceptual Framework for Describing the Phenomenon of New Venture Creation. Academy of Management Review, vol. 10 (4) pp. 696-706.

Jorge, C. (2017). Tarea pendiente . Vistazo, 310-312.

Juan Fernando Reinoso Lastra \& Mario Enrique Uribe Macías. (2013). Emprendimiento y Empresarismo. Bogotá, Colombia: Ediciones U.

London Business School. (2005). Global Entrepreneurship Monitor Perú 2004-2005. Lima: Esan.

Malagón, F. A. (2003). ¿Cómo orientar el espíritu emprendedor hacia la creación de empresas? Revista escuela de administración de negocios, 72-81.

Mises, L. v. (1961). Socialismo: análisis económico y sociológico. México: Editorial Hermes.

Pérez, Pilar \& Oliver, Rúben \& Merritt, Humberto \& Márquez, Alejandro \& León, Jorge. (2006). El emprendedor en México: ingenio vs innovación. I congreso Iberoamericano de Ciencia, Tecnología, Sociedad e Innovación CTS + I, 1-13.

Sabbagh Aarón, Mackinlay Matías. (2011). El método de la innovación creativa. Buenos Aires: Granica.

Schumpeter, J. (1982). Historia del análisis económico 2da. Edición. Madrid: Arial.

Valencia, E. (2017). Inovar es cosa de líderes. Vistazo, 302-304.

Vicepresidencia de la República del Ecuador. (Marzo de 2015). Estrategia Nacional para el cambio de la matriz productiva. Obtenido de file://C:/Users/Tany/Desktop/Articulos/Emprendimiento/Matriz\%20productiva.pdf 